\title{
Cumplimiento de garantía de oportunidad GES en ayudas técnicas en una comuna urbana de la Región Metropolitana
}

\author{
MAURO TAMAYO ${ }^{(1)}$ y FERNANDA ALEITTE(1)
}

\section{RESUMEN}

Objetivo: El sistema de garantías explícitas en salud (GES) considera problemas prioritarios de salud. El objetivo es evaluar el cumplimiento de la garantía de oportunidad en la entrega de ayudas técnicas (AT) en tres centros de salud de una comuna urbana de la Región Metropolitana. Material y método: Estudio no experimental de tipo descriptivo, que utiliza medidas de frecuencia, mediana, percentil 25-75 y amplitud intercuartil. Los datos fueron analizados con el programa SPSS 17.0 para Windows, con Kruskal-Wallis para establecer diferencias en el nivel de cumplimiento entre los centros de salud $(p<0.05)$. Se incluyeron las prestaciones registradas en Sigges entre el 1 de enero de 2007 hasta el 13 de enero de 2011. Según el tipo de AT, la muestra fue organizada en 2 grupos, prestaciones con plazo de 20 días $(n=473)$ y el grupo 2 con plazo de 90 días $(n=406)$. Resultados: De las prestaciones correspondientes al grupo $N^{\circ} 1$, sólo el $45.7 \%$ fue entregada dentro de los plazos que establece GES. Por su parte, el grupo $N^{\circ} 2$ presenta un porcentaje de cumplimiento de $68.7 \%$. Conclusiones: El porcentaje de cumplimiento de la garantía de oportunidad fue para el primer grupo menor a la mitad de las prestaciones y en el segundo grupo si bien mejora, casi en un tercio de ellas no se cumple la garantía de oportunidad en las AT en los plazos establecidos por ley.

Palabras clave: GES, garantía de oportunidad, ayudas técnicas, reforma de salud.

\section{ABSTRACT}

COMPLIANCE WITH PUNCTUALITY GUARANTEES (GES) FOR TECHNICAL ASSISTANCE IN AN URBAN COUNTY OF THE METROPOLITAN REGION

Objective: The system of explicit health guarantees (GES) covers priority health problems. The objective of the study was to evaluate compliance with the guarantee of punctuality of technical assistance in three health centers in an urban community in the Metropolitan Region. Materials and methods: Non experimental descriptive study, which used measures of frequency, median, 25-75 percentile and interquartile range. The data was analyzed with SPSS 17.0 for Windows, with Kruskal-Wallis to establish differences in compliance among health centers $(p<0.05)$. All technical assistance provisions in Sigges between January 1, 2007 until January 13, 2011 were included. According to the type of technical assistance, the sample was organized in two groups, group 1, assistance with a time limit of 20 days $(n=473)$ and group 2 , assistance with a limit of

(1) Línea de Gestión y Salud Pública. Escuela de Kinesiología. Facultad de Medicina. Universidad de Chile. Independencia 1027. Santiago. Chile. mtamayo@med.uchile.cl 
90 days $(n=406)$. Results: Of all assistance from group 1, only 45.7\% were fulfilled within the time limits established by GES. In group 2, compliance was $68.7 \%$.

Conclusions: The percentage of compliance with the guarantee of punctuality for the first group accounted for less than half of all assistance in that group, and although compliance was better in the second group, almost a third of all assistance in that group did not comply with the time limits established by law.

Key words: GES, puntuality guarantee, technical assitance, health reform.

\section{INTRODUCCIÓN}

Chile enfrenta un proceso de envejecimiento acelerado. Actualmente la población mayor de 65 años se aproxima al millón y medio de habitantes, y se espera un aumento significativo en los próximos años ${ }^{1,2}$. Según los resultados del censo 2002, la población total de la comuna de Cerro Navia alcanzaba 148.312 habitantes, de los cuales 10.234 (6.9\%) correspondía a personas mayores de 65 años. En la actualidad la población inscrita en los centros de salud primaria y validada por Fonasa para 2011, es de 139.176 personas; de éstos 15.737 corresponden a personas sobre 65 años $^{3,4}$. Situación que tiene importantes implicancias sobre el sistema de salud, pues el particular perfil de morbilidad y discapacidad de este grupo etario, requiere orientar las estrategias sanitarias a la mantención y recuperación de la funcionalidad, autonomía e integración social, implementando beneficios específicos para los adultos mayores que les permitan tener un envejecimiento activo ${ }^{5,6}$ previniendo la discapacidad y mejorando su calidad de vida ${ }^{7-9}$. Pues, por sobre el deterioro físico, la interacción del adulto mayor con su entorno es lo que definirá su autonomía ${ }^{5,9}$. Los adultos mayores autónomos y activos constituyen un capital social, muy importante para los próximos decenios ${ }^{5,10}$.

La salud es un derecho fundamental de las personas, y recae en el Estado el rol de garantizarlo, generando condiciones en las cuales todos puedan vivir lo más saludablemente

Tabla 1- Descripción de la muestra.

\begin{tabular}{lcccc}
\hline \multicolumn{1}{c}{ Centros de salud } & Dr. Steeger & Dr. Albertz & Cerro Navia & Total \\
Mujeres & 245 & 161 & 164 & 570 \\
Hombres & 94 & 75 & 104 & 273 \\
Total & 339 & 236 & 268 & 843 \\
Edad (años) & $($ media $\pm \mathrm{DE})$ & $($ media $\pm \mathrm{DE})$ & $($ media $\pm \mathrm{DE})$ & $($ media $\pm \mathrm{DE})$ \\
Mujeres & $78.29 \pm 7.42$ & $81.75 \pm 7.7$ & $80.98 \pm 8.4$ & $80.04 \pm 7.93$ \\
Hombres & $78.43 \pm 7.44$ & $80.68 \pm 7.05$ & $77.47 \pm 8.38$ & $78.68 \pm 7.8$ \\
Previsión & & & & \\
Fonasa A & 141 & 71 & 135 & 347 \\
Fonasa B & 172 & 153 & 112 & 437 \\
Fonasa C & 13 & 5 & 8 & 26 \\
Fonasa D & 12 & 6 & 13 & 31 \\
No especificado & 1 & 1 & 0 & 2 \\
Total Prestaciones & 365 & 256 & 305 & 926 \\
$\quad$ Grupo 1 & 175 & 137 & 161 & 473 \\
Grupo 2 & 171 & 102 & 133 & 406 \\
\hline
\end{tabular}

DE (Desviación Estándar)

Grupo 1: Bastones, colchones y cojines

Grupo 2: Sillas de ruedas y andadores 
posible ${ }^{11-13}$. Chile cuenta con un régimen de garantías sanitarias: Garantías Explícitas en Salud (GES), en el cual se garantiza acceso universal, protección financiera, oportunidad y calidad a todos quienes posean una condición contemplada en el programa, bajo el criterio de priorizar aquellas enfermedades de mayor impacto sanitario $^{14-20}$. Dentro de éstas, la garantía de oportunidad establece un plazo máximo en el cual deberán ser atendidas ${ }^{14-17}$.

El sistema GES comprende variados programas dirigidos a los adultos mayores, entre los que se encuentra la entrega de órtesis o ayudas técnicas (AT) a personas de 65 años o más que lo requieran según indicación médica. Ésta indicación debe considerar el ciclo completo, incorporando en este proceso a médicos(as) y a kinesiólogos(as), quienes en conjunto evaluarán y seleccionarán la AT más apropiada, luego el kinesiólogo se encargará de la educación referente al buen uso de la AT, entrenando al beneficiario durante 2 sesiones, posteriormente, éste debe ser monitoreado, ya que la entrega de AT constituye un aporte importante a la autonomía, calidad de vida y participación de las personas que las reciben. Las AT garantizadas por el GES son: bastones, cojín y colchón anti-escaras, los cuales serán entregados en un plazo máximo de 20 días desde la indicación, además de andadores y sillas de ruedas que serán entregados dentro de 90 días desde la indicación ${ }^{21}$. La responsabilidad de determinar el requerimiento de AT, su entrega y la atención educativa por parte del kinesiólogo(a) es de todos los establecimientos de la red, en sus diferentes niveles, los cuales se coordinan a través del Sistema de Información para la Gestión de las Garantías Explícitas de Salud (Sigges) ${ }^{22}$, no obstante lo anterior por la amplia cobertura de APS, es la principal responsable de esta garantía.

La Superintendencia de Salud se encarga de fiscalizar el cumplimiento de las garantías $\mathrm{GES}^{14,15}$, sus resultados evidencian un alto porcentaje de incumplimiento de la garantía de oportunidad ${ }^{15}$. En los datos publicados en

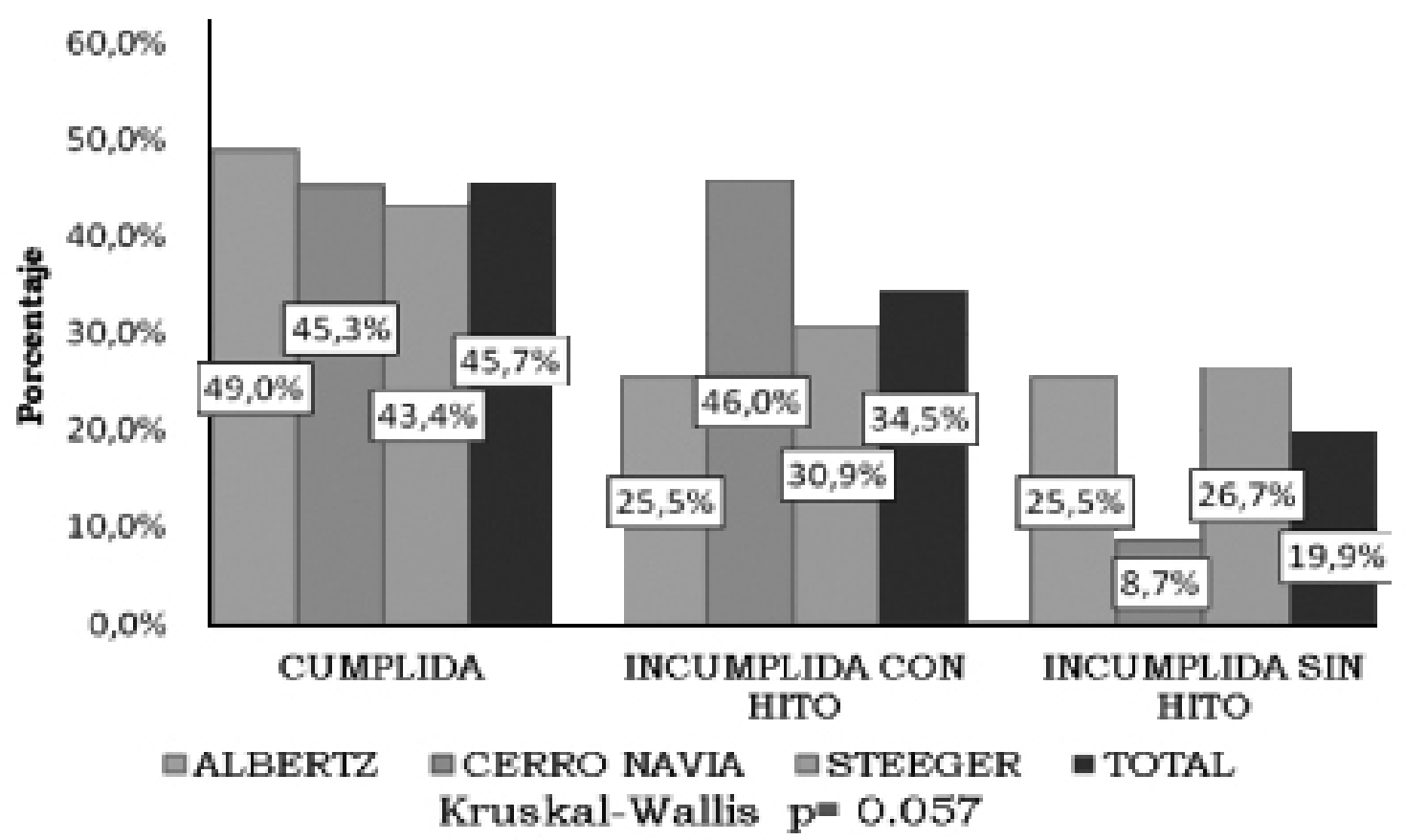

Figura 1. Cumplimiento garantía de oportunidad ayudas técnicas del grupo 1 (bastones, colchones y cojines) por centro de salud y total $(n=473)$. 


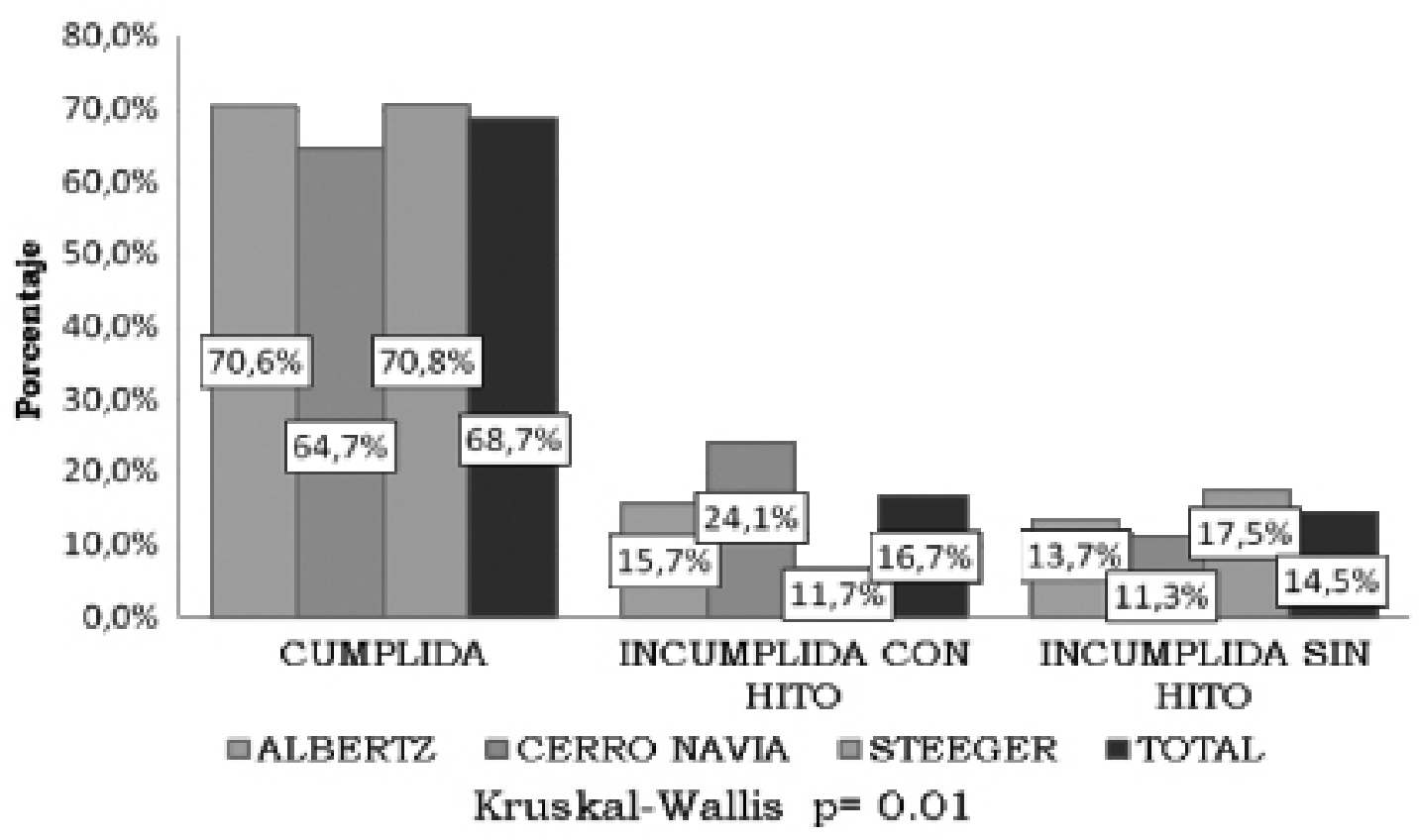

Figura 2. Cumplimiento garantía de oportunidad ayudas técnicas del grupo 2 (sillas de ruedas y andadores) por centro de salud y total $(n=406)$.

el año 2007 se observa un $19.93 \%$ de retrasos en la entrega de $\mathrm{AT}^{15}$ mientras que para 2009 éste llega a $15 \%{ }^{23}$, sobrepasando los 120 días de retraso en algunos casos. En el último registro del año 2010, la entrega de AT presenta el cuarto mayor porcentaje de retrasos sobre el total de garantías ${ }^{24}$.

El objetivo de este estudio es evaluar el cumplimiento de la garantía de oportunidad en el programa GES de entrega de Ayudas Técnicas a personas de 65 años o más, en tres centros de salud de la comuna de Cerro Navia, Región Metropolitana.

\section{MATERIAL Y MÉTODO}

El presente trabajo es un estudio no experimental de tipo descriptivo. La muestra del estudio considera la totalidad de las prestaciones pertenecientes a la población de adultos mayores de 65 años o más, incorporados al programa y registrado en el Sigges, entre el 1 de enero de 2007 hasta el 13 de enero de 2011, en 3 centros de salud de la comuna de Cerro Navia. Se excluyó de este estudio al Cesfam Lo Amor por no cumplir con el criterio de temporalidad pues inicia su funcionamiento el año 2009. A partir de cada registro se recopiló un total de 926 prestaciones, de las cuales fueron excluidas las prestaciones que al $13 \mathrm{de}$ enero del 2011 aún estaban dentro del plazo de cumplimiento $(n=43)$ y aquellas con datos incompletos o con errores de digitación $(n=4)$, por tanto la muestra quedó constituida por 879 prestaciones, pertenecientes a 843 adultos mayores. La diferencia se debe a que algunas de estas personas requieren más de una AT. Según el tipo de AT a entregar, la muestra fue organizada en 2 grupos, el primero se constituye de aquellas con plazo de 20 días $(n=473)$, y el grupo 2, con plazo de 90 días $(\mathrm{n}=406)$.

La información obtenida por cada paciente fue: edad, género, grupo de AT, fecha de ingreso, fecha de entrega de la prestación (en aquellas que correspondía), fecha de plazo máximo. Con estos datos la muestra fue clasificada 


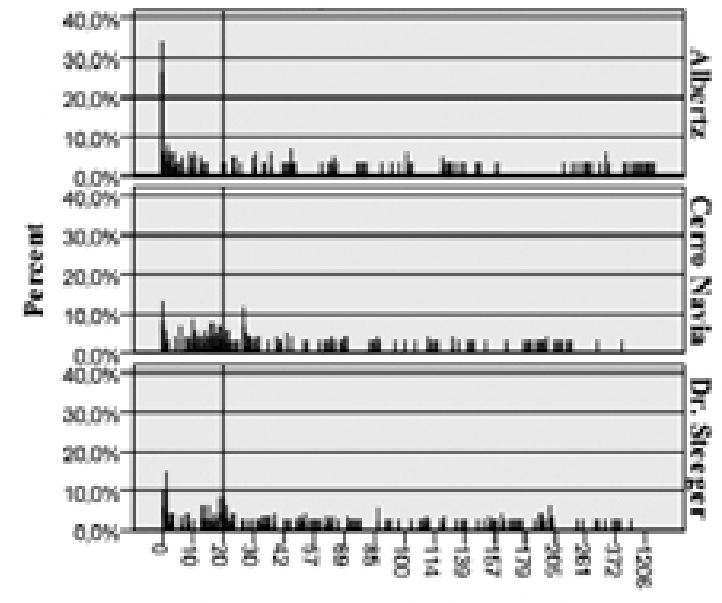

Tiempo de espera Grupo 1

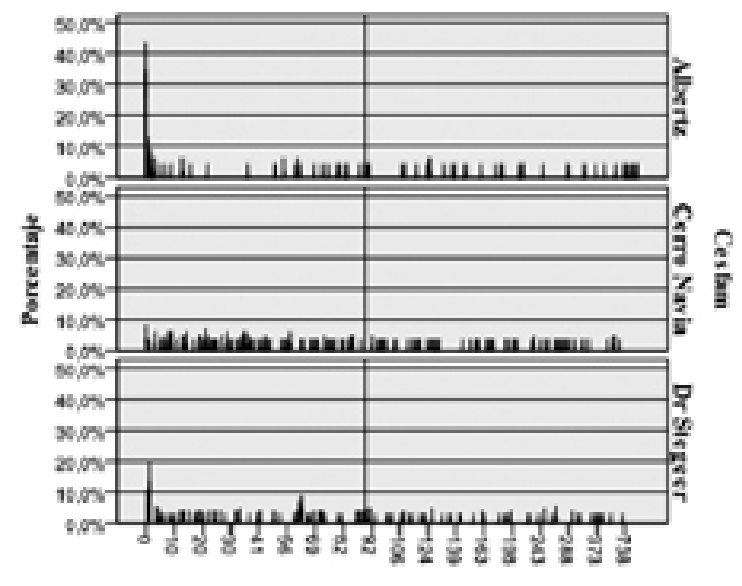

Tiempo de espera Grupo 2

Línea vertical representa plazo máximo de garantía de oportunidad GES.

Grupo 1: Bastones, colchones y cojines

Grupo 2: Sillas de ruedas y andadores

Figura 3. Tiempo de entrega y espera de Ayudas Técnicas del grupo $1(\mathrm{n}=474)$ y grupo 2 ( $\mathrm{n}=406)$ por centro de salud.

en 3 estados:

- Cumplida: la prestación fue otorgada dentro de los plazos establecidos en la legislación vigente.

- Retrasada con hito: la prestación fue otorgada fuera de los plazos establecidos en la legislación vigente.

- Retrasada sin hito: aquella prestación que encontrándose vencidos los plazos, no fue entregada al beneficiario/ durante el tiempo de seguimiento.

En base a esto se realiza un análisis de frecuencia para estimar el porcentaje de cumplimiento de cada grupo de AT y centro de salud. El tiempo de espera es analizado por grupo de prestación y centro de salud a través de mediana, percentil 25-75 y amplitud intercuartil. Los datos fueron analizados con el programa SPSS 17.0 para Windows con la prueba Kolmogorov-Smirnov para evaluar la distribución de la muestra y la prueba Kruskal-Wallis para establecer diferencias entre los centros de salud evaluados con una significancia de $\mathrm{p}<0.05$.

\section{RESULTADOS}

La descripción de la muestra está expresada en la Tabla 1. La prueba Kolmogorov-Smirnov indica que la muestra no presenta una distribución normal, por lo cual los resultados serán analizados con pruebas no paramétricas.

En un análisis de la garantía de oportunidad en la entrega de AT en ambos grupos, encontramos que de las 879 prestaciones 495 $(56.3 \%)$ fueron entregadas dentro del plazo establecido por la ley, mientras que las 384 restantes $(43.7 \%)$ se encuentran retrasadas. De esta última se desprenden 2 subcategorías: Retrasadas con hito $(26.3 \%)$ y Retrasadas sin hito (17.4\%), encontrando diferencias significativas entre cada estado $(\mathrm{p}=0.01)$. El análisis de cada grupo de AT puede observarse en las Figuras 1 y 2.

\section{Grupo 1: Bastones, colchones y cojines}

Del total de prestaciones de este grupo, 175 (36.9\%) pertenecen al Cesfam Dr. Steeger, 137 (28.9\%) al Cesfam Albertz, y 161 (34.0\%) al 
Cumplimiento de garantía de oportunidad GES en ayudas técnicas en una comuna urbana - Mauro Tamayo et al

Tabla 2 - Tiempo espera para la entrega de ayudas técnicas ambos grupos (Mediana, amplitud intercuartil, percentil 25-75 y tiempos máximos).

\begin{tabular}{lcccccccc}
\hline & \multicolumn{3}{c}{ Grupo 1: Bastones, colchones y cojines } & \multicolumn{3}{c}{ Grupo 2: Sillas de ruedas y andadores } \\
\hline $\begin{array}{l}\text { Centro de } \\
\text { Salud }\end{array}$ & $\begin{array}{c}\text { Mediana } \\
\text { (días) }\end{array}$ & $\begin{array}{c}\text { Amplitud } \\
\text { intercuartil } \\
\text { (días) }\end{array}$ & $\begin{array}{c}\text { Percentil } \\
25-75\end{array}$ & $\begin{array}{c}\text { Máximo } \\
\text { (días) }\end{array}$ & $\begin{array}{c}\text { Mediana } \\
\text { (días) }\end{array}$ & $\begin{array}{c}\text { Amplitud } \\
\text { intercuartil } \\
\text { (días) }\end{array}$ & $\begin{array}{c}\text { Percentil } \\
25-75 \\
\text { (días) }\end{array}$ & $\begin{array}{c}\text { Máximo } \\
\text { (días) }\end{array}$ \\
Dr. Albertz & 24 & 101 & $0-101$ & 1234 & 11 & 124 & $0-124$ & 1364 \\
Cerro Navia & 24 & 57 & $12-68.5$ & 548 & 57 & 105 & $21,5-126$ & 537 \\
Dr. Steeger & 34 & 97 & $13-110$ & 1067 & 62 & 104 & $6-110$ & 570 \\
Total & 27 & 86 & $8-93$ & 1234 & 57 & 111 & $6-117$ & 1364 \\
\hline
\end{tabular}

Plazo mínimo en ambos grupos: 0 días.

Cesfam Cerro Navia. Al evaluar el porcentaje de cumplimiento de la garantía de oportunidad (GO) por cada centro, encontramos que los Cesfam Albertz, Cerro Navia y Dr. Steeger entregaron un $49 \%, 45.3 \%$ y $43.4 \%$, respectivamente, de sus prestaciones dentro del plazo establecido (20 días). Por tanto existe un porcentaje de incumplimiento de $51 \%$ para el Cesfam Albertz, un 54.7\% para el Cesfam Cerro Navia y un $56.6 \%$ para el Cesfam Dr. Steeger. La distribución de aquellas que se entregaron fuera de plazo y aquellas que, a la fecha de corte, aún no se entregaban, se observa en la Figura 1. Las diferencias en el nivel de cumplimiento entre los centros resultan no ser estadísticamente significativas $(p=0.057)$. En promedio, los tres Cesfam presentan un 24\% de AT con más de 100 días de espera.

\section{Grupo 2: Sillas de ruedas y andadores}

Del total de prestaciones del grupo 2, 102 (25.12\%) pertenecen al Cesfam Albertz, 133 $(32.76 \%)$ al Cerro Navia y $171(42.12 \%)$ al Steeger (Tabla 1).

Sobre el porcentaje de cumplimiento de la GO por cada centro, los Cesfam Albertz, Cerro Navia y Steeger entregaron un $70.6 \%$, $64.6 \%, 70.8 \%$, respectivamente, de sus prestaciones dentro del plazo establecido (90 días). Por tanto existe un porcentaje de retraso de $29.4 \%$ para el Cesfam Albertz, un 35.3\% para el Cesfam Cerro Navia y un $29.2 \%$ para el Cesfam Steeger. De este porcentaje de retraso se desprenden 2 categorías (Retrasadas con hito y Retrasadas sin hito), su frecuencia queda establecida en la Figura 2. Se observan diferencias significativas en el cumplimiento de los centros en este grupo $(\mathrm{p}=0.01)$.

En la Tabla 2 se observan los resultados referentes al tiempo de espera para ambos grupos de AT clasificadas por Cesfam. La frecuencia de entrega por cada centro queda expresada en la Figura 3, graficando su evolución durante el tiempo analizado.

\section{DISCUSIÓN}

Investigar en relación al nivel de cumplimiento de las diferentes garantías que establece GES debe convocar mayores esfuerzos. Como señala González, "se requiere de un trabajo mancomunado, coordinado, profesional y dedicado de todos los actores que puedan jugar algún papel en la planificación, implementación, puesta en marcha, operación y evaluación del Plan" ${ }^{25}$. Los requerimientos de diferentes ayudas técnicas en nuestra población con creciente sobrevida, deberá atenderse con preocupación y determinación. La comuna de Cerro Navia presenta importante vulnerabilidad social ${ }^{26}$, lo cual obliga a los diferentes actores a realizar una labor cada vez de mayor coordinación y programación en el cumplimiento de sus diferentes acciones.

En esta investigación debe significar especial preocupación que en el grupo de ayudas vinculadas a bastones, colchones y cojines, en promedio, los diferentes centros no son capa- 
ces de cumplir en los plazos establecidos en la Ley 19.864, ni siquiera para la mitad de las prestaciones; esto es concordante con otros estudios de cumplimiento de protocolo $\mathrm{GES}^{27,28}$.

Se observan mejores porcentajes de cumplimiento en la entrega de AT del grupo $\mathrm{N}^{\circ} 2$, sillas de ruedas y bastones, en comparación con el grupo $\mathrm{N}^{\circ} 1$, con una diferencia de $23 \%$ entre ellos. Esto es contradictorio al pensar que son ayudas técnicas de mayor costo con relación a los bastones, cojines y colchones antiescaras. En la actualidad, la adquisición de las diferentes AT para ésta comuna se realiza a través del Servicio de Salud Metropolitano Occidente, lo cual resta autonomía a la gestión local, pero llama la atención los disímiles resultados entre centros.

En ambos grupos de AT se observa que el Cesfam Albertz entrega, al menos, el 25\% de sus AT el mismo día de la inscripción (0 días de espera). Sin embargo, el máximo tiempo de espera de este Cesfam supera con creces los demás centros (más de 3 años de espera), lo cual se ve reflejado en una gran amplitud intercuartil, que podría evidenciar una estrategia que prioriza a los nuevos beneficiarios, postergando a las personas en espera. Respecto al Cesfam Cerro Navia, se aprecia una mayor concentración de entrega alrededor de la fecha límite. Al observar el tiempo de espera máximo en ambos grupos de prestaciones, este centro presenta los valores más bajos, destacando con los valores más bajos del grupo 1 de AT en el percentil 75 y la amplitud intercuartil, lo cual reflejaría la prioridad de entregar las AT en orden de inscripción. El Cesfam Steeger entrega aproximadamente el $10 \%$ de las AT del grupo 1 y el $20 \%$ de las AT del grupo 2 el mismo día de inscripción; luego, la entrega es constante y uniforme en el tiempo.

Cabe destacar que casi un tercio de las AT del grupo 1 son entregadas después de los 100 días de espera, 5 veces más de lo que establece la $\mathrm{GO}$, lo cual se relaciona con el alto tiempo de espera máximo que presenta.

Un informe de la Superintendencia de Salud detalla que la entrega de AT se encuentra dentro de las 10 prestaciones GES con mayor retraso de la GO. En el mismo informe se observa que el Servicio de Salud Metropolitano Occidente ocupa el cuarto lugar entre los centros con mayor retraso de la GO; del total de prestaciones $\mathrm{GES}^{29}$, lo cual concuerda con nuestros resultados.

En un estudio en 2007, se observa que este Servicio no cumple con la GO de 191 pacientes en la entrega de AT del grupo 1, presentando un tiempo de espera máximo de 150 días, 7.5 veces mayor al plazo establecido para la $\mathrm{GO}$, respecto al grupo 2, no se cumple la GO de 183 pacientes, con un máximo de tiempo de espera de 126 días, alrededor de un mes de retraso $^{30}$, cifras significativamente menor a los valores arrojados en esta investigación.

En lo inmediato, si queremos mejorar la calidad de los servicios de salud deberemos, en vez de redestinar los escasos recursos financieros disponibles, aumentarlos en cantidad suficiente, al menos para alcanzar las metas propuestas inicialmente ${ }^{25}$. A raíz de estos resultados se requiere un análisis acabado del proceso de gestión de la red asistencial, los procedimientos, recursos y costos asociados por el no cumplimiento de las garantías.

De acuerdo al último Estudio nacional de la dependencia en las personas mayores ${ }^{31}$, en Chile, el 14,7\% de quienes tienen más de 60 años viven solos (247.797 adultos mayores), presentando un aumento de 5 puntos porcentuales respecto al Censo 1992. Vivir solo puede generar condiciones de vulnerabilidad, sugiere desprotección frente a situaciones relacionadas con el deterioro biológico inherente al envejecimiento, entre ellas la dificultad de desplazamiento y autocuidado, generan un importante impacto en las actividades de la vida diaria, determinando algún grado de disfuncionalidad. La entrega de AT se enmarca en la labor de recobrar la autonomía del adulto mayor y aporta, en cierto grado, independencia. Hacer frente a los cambios del envejecimiento, y dentro de éstos lograr la mayor independencia funcional en los adultos mayores, es una tarea de trascendental importancia para los próximos años. Por ello, es indispensable objetivar nuestros avances para contar con una base de 
políticas públicas que nos asegure una mejor calidad de vida.

\section{AGRADECIMIENTOS}

A todo el equipo humano de Cerro Navia, en especial a la Dirección de Salud, directores(as) de centros y a los encargados del Registro Sigges local, quienes nos ayudaron en la recolección de datos necesarios para llevar a cabo este estudio.

Esta investigación no contó con financiamiento externo.

\section{REFERENCIAS}

1. ONU (United Nations). World Population Prospects: The 2006 Revision Department of Economic and Social Affairs, Population Division. New York; 2007 [Fecha de acceso: 3 de Enero 2011] URL disponible en: http://www.un.org/esa/population/publications/ wpp2006/WPP2006_Highlights_rev.pdf

2. INE. Enfoque estadístico - adulto mayor - Boletín Informativo del Instituto Nacional de Estadísticas; Septiembre 2010 [Fecha de acceso: 3 enero 2011] URL disponible en: http://www.ine.cl//filenews/ files/2011/septiembre/pdf/adulto_mayor_web_0109-10(1).pdf

3. http://www.ine.cl/canales/chile_estadistico/estadisticas_sociales_culturales/adultosmayores/pdf/mayorescenso2002.pdf

4. DIRECCIÓN DE SALUD. Corporación de Desarrollo Social de Cerro Navia. Plan de Salud 20112012.

5. SENAMA. Gobierno de Chile. Política Nacional para el Adulto Mayor (actualización agosto 2004 [Fecha de acceso: 3 de Enero 2011] URL disponible en: http://www.senama.cl/Archivos/720.pdf

6. OMS/WHO. $58^{\circ}$ Asamblea Mundial de la Salud WHA58.16. Promoción de un envejecimiento activo y saludable. Novena sesión plenaria, 25 de mayo de 2005 [Fecha de acceso.3 de enero 2011 ] URL disponible en: http://www.who.int/mediacentre/news/ releases/pr72/es/index.html

7. RODRÍGUEZ M. Envejecimiento y gasto en salud. Fonasa; octubre 2009 [Fecha de acceso: 23 de diciembre 2010] URL disponible en: www.edac.cl/celade/ noticias/paginas/2/37482/M Isabel Rodriguez.pdf

8. CEPAL. Redes de apoyo social de las personas mayores en América Latina y el Caribe. Celade. Santiago; diciembre 2000.
9. CHAWLA T. The participation of the elderly in development. United Nations, CSDHA, International Seminar Expert Group. 1998.

10. ENGLER T, PELÁEZ M. Aged-southern cone of south america - social conditions. Inter-American Development Bank. Panamerican Health Organization. Washington DC; 2002.

11. OMS (WHO) Constitución de la Organización Mundial de la Salud. El derecho a la salud [Fecha de acceso: 30 diciembre de 2010] URL disponible en: http://www.who.int/mediacentre/factsheets/fs323/ es/index.htlm

12. Declaración de Alma Ata, Conferencia Internacional de Atención Primaria de Salud, Alma-Ata, URSS, 6-12 septiembre 1978 [Fecha de acceso: 3 de enero 2011] URL disponible en: http://www.inclusion-ia. org/espa\%F1ol/Norm/AlmaAta-02-1.pdf http:// www.alma-ata.es/declaraciondealmaata/declaraciondealmaata.html

13. FRENZ P. Indicadores para evaluar el derecho a la salud en Chile [Tesis Magister]. Santiago: Servicio de Publicaciones, Universidad de Chile; 2009.

14. 14. Ley 19.966 - Del Régimen General de Garantías en Salud. Publicada el 03/09/2004 [Fecha de acceso 22 de diciembre 2010] URL disponible en. http:// www.leychile.cl/Navegar?idNorma $=229834 \& \mathrm{r}=1$

15. BARRÍA MS. Auge 2005-2008-Implementación de garantías explícitas en salud. Programa Contigo mejor Salud; Agosto 2008. Ministerio de Salud (Minsal). [Fecha de acceso: 23 diciembre 2010] URL disponible en: http://www.pos.gov.co/Documents/ Jornada\%20Intercambio/GES_FINAL.pdf

16. ZÚÑIGA A. Sistema de salud sanitario y Reforma Auge en Chile. Acta Bioethica 2007; 13 (2)

17. MINISTERIO DE SALUD. Información para el usuario. [Fecha de acceso 22 de Diciembre de 2010] URL disponible en: http://augeenlinea.fonasa.cl/portal

18. VALDIVIESO V., MONTERO J. El plan Auge: 2005 al 2009. Rev. méd. Chile, Ago 2010, vol.138, no.8, p.1040-1046.

19. ROMAN O., MUÑOZ F. Una mirada crítica en torno al plan Auge. Algunos aspectos generales y valóricos. Rev. méd. Chile, Dic 2008, vol.136, no.12, p.1599-1603.

20. CRUZ COKE R. Cobertura de patologías en Plan Auge de Chile. Rev. méd. Chile, Ago 2007, vol.135, no.8, p.1082-1083.

21. MINISTERIO DE SALUD Guía Clínica de órtesis para personas de 65 años y más [Fecha de acceso: 21 diciembre 2010] URL disponible en: http://www. redsalud.gov.cl/archivos/guiasges/ortesisr.pdf

22. MINISTERIO DE SALUD. Redes de atención Auge. Sistema Nacional de Servicios de Salud, De- 
partamento GES de Redes Asistenciales, Subsecretaría de Redes Asistenciales, División de Gestión de Redes Asistenciales. Diciembre 2010.

23. SUPERINTENDENCIA DE SALUD. Minuta Técnica. Fiscalización garantía de oportunidad en personas beneficiarias de Fonasa. Enfoque sistémico y por Riesgo - Regiones 2009. [Fecha de acceso. 23 de Diciembre 2010] Disponible en: http://www. supersalud.cl/documentacion/569/articles-6160_recurso 1.pdf

24. SIGGES FONASA. Información garantías de oportunidad acumulado al 30 noviembre 2010. Fonasa. Gobierno de Chile [Fecha de acceso: 3 de Enero de 2011] URL disponible en http://www.fonasa.cl/ prontus_fonasa/site/artic/20101210/asocfile/director_go al_30_nov_2010_oficial.doc

25. GONZÁLEZ F. Là implementación del Plan de Acceso Universal y Garantías Explícitas (Plan Auge) ha deteriorado la calidad del tratamiento de pacientes con insuficiencia renal terminal. Rev. méd. Chile, Oct 2006, vol.134, no.10, p.1288-1294.

26. MINISTERIO DEL INTERIOR. División de Seguridad Pública, Índice de Vulnerabilidad Social y Delictual 2009 [Fecha de acceso 22 de Junio de 2011] URL disponible en. http://www.productoprotegido.cl/estadisticas/Indice_de_Vulnerabilidad_Social_2009.pdf
27. NAZZAL C. et al. Impacto del Plan Auge en el tratamiento de pacientes con infarto agudo al miocardio con supradesnivel ST, en hospitales chilenos. Rev. méd. Chile, Oct 2008, vol.136, no.10, p.1231-1239.

28. WANG Y. et al. Análisis del cumplimiento de la Guía Clínica del Auge en pacientes con accidente cerebrovascular isquémico: la utilización de tecnología sanitaria de diagnóstico para prevención secundaria en un hospital público. Rev. méd. Chile, Jun 2011, vol.139, no.6, p.697-703.

29. MINISTERIO DE SALUD. Información garantías de oportunidad acumulado al 30 de marzo del 2011. [Fecha de acceso: 3 de Julio 2011] URL disponible en: http://www.redsalud.gov.cl/portal/docs/page/ minsalcl/g_general/elementos/archivos/inf.garantias310311.pdf

30. MANAGEMENT. Listas de espera Auge y No Auge en servicios de salud a nivel nacional 2007. [Fecha de acceso 24 de Marzo 2011] URL disponible en: www.alturamanagement.cl/Estudios/Listas_de_Espera2.doc

31. SENAMA Estudio nacional de la dependencia en adultos mayores 2010 [Fecha de acceso: 25 Junio de 2011]URL disponible en http://www.senama.cl/ archivos/estudiodependencia.pdf

Recibido: 11 de julio de 2011 Aprobado: 16 de noviembre de 2011

Usted puede comentar éste y otros artículos publicados en la Revista Chilena de Salud Pública, enviando un correo electrónico a revistasp@med.uchile.cl 